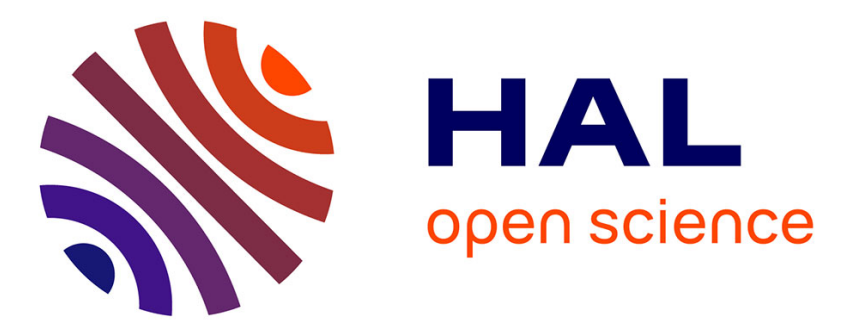

\title{
ÉTUDE EXPÉRIMENTALE DE LA DIFFRACTION ACOUSTIQUE PAR UNE CIBLE RIGIDE : CYLINDRE TERMINÉ PAR DEUX HÉMISPHÈRES
}

R. Hazebrouck

\section{- To cite this version:}

R. Hazebrouck. ÉTUDE EXPÉRIMENTALE DE LA DIFFRACTION ACOUSTIQUE PAR UNE CIBLE RIGIDE: CYLINDRE TERMINÉ PAR DEUX HÉMISPHÈRES. Journal de Physique IV Proceedings, 1992, 02 (C1), pp.C1-705-C1-708. 10.1051/jp4:19921152 。 jpa-00251111

HAL Id: jpa-00251111

https://hal.science/jpa-00251111

Submitted on 1 Jan 1992

HAL is a multi-disciplinary open access archive for the deposit and dissemination of scientific research documents, whether they are published or not. The documents may come from teaching and research institutions in France or abroad, or from public or private research centers.
L'archive ouverte pluridisciplinaire HAL, est destinée au dépôt et à la diffusion de documents scientifiques de niveau recherche, publiés ou non, émanant des établissements d'enseignement et de recherche français ou étrangers, des laboratoires publics ou privés. 


\section{ÉTUDE EXPÉRIMENTALE DE LA DIFFRACTION ACOUSTIQUE PAR UNE CIBLE RIGIDE : CYLINDRE TERMINÉ PAR DEUX HÉMISPHÈRES}

\section{R. HAZEBROUCK}

Laboratoire d'Acoustique, Université de Lille Flandres Artois, Antenne de Béthune, nue du Moulin à Tabac, F-62408 Béthune cedex, France

\footnotetext{
Abstract: This study was carried out with the aim of bringing some experimental results to valid theorical results [1] computed with programmes using the 'Geometrical Theory of Diffraction' [2] .

A bistatic geometry is used, a broadband sphérical impulsive wave impinges on the rigid target in air. At a distance $r=10 a$ (a: characteristic parameter of the target), the signal of the diffracted wave is measured.

After signal processing ( time averaging, time filtering,FFT,...) one can observe, for several geometries:

-The signal of the diffracted wave:

It consists of the reflected wave and also of a main creeping wave which can be shown. For particular geometries, a secondary creeping wave coming from the hemispheres and a weak echo due to the discontinuity of curvature can be isolated.

-The frequency responses:

They are carried out for $k a \leq 15, \theta \in\left[10^{\circ}, 180^{\circ}\right]$; from these, diagrams of scattering of sound are deduced.

The time filtering enables us to get the 'reflection coefficient of the target'.

Analysis of the results shows the importance of creeping waves and also of echos dues to the discontinuity in curvatuve.
}

\section{INTRODUCTION.}

La diffraction acoustique d'une onde plane monochromatique par le corps rigide LINE (cylindre limité par deux hémisphères) a été calculée dans le cadre de la Théorie Géométrique de la Diffraction $[1,2]$.

L'étude expérimentale présentée ici est menée dans l'air en bistatique et en régime impulsif. Elle permet de proposer une attribution aux différents échos observés dans le signal diffracté par 'IINE rigide' donnant ainsi une idée de 1'importance relative des phénomènes pour la géométrie considérée. Les réponses en fréquences mesurées pour chaque géométrie couvrent un domaine $X=k a \leq 15$ (a:rayon du cylindre; k: nombre d'onde) et permettent d'établir des diagrammes de rayonnement. Le filtrage de l'onde réfléchie conduit à l'établissement de diagrammes du 'coefficient de réflexion' de la cible.

\section{CONDITIONS EXPERIMENTALES, PRINCIPE DE LA MESURE:}

La source impulsive $S$ et le microphone $M$ sont placés dans le plan équatorial de la cible maintenue verticalement, avec $r_{\circ}=1,5 \mathrm{~m} ; \mathrm{a}=0,034 \mathrm{~m}, \mathrm{r} / \mathrm{a}=10^{\circ}$ et $\theta \in\left[10^{\circ}-180^{\circ}\right]$ (figure 1). Les trois cibles étudiées $\left(n^{\circ} 1: L / a=3 ; n^{\circ} 2: L / a=5 ; n^{\circ} 3: L / a=12\right.$; L: longueur totale de la cible) sont en aluminium et se comportent, dans $l$ 'air, comme des corps parfaitement rigides. L'onde émise est sphérique et, pour cette géométrie, l'angle limite d'ombre $\left(\theta 1\right.$ i $\left.m=A r c c o s(a / r)+\operatorname{Arccos}\left(a / r_{0}\right)\right)$ est égal à $173^{\circ}$. 


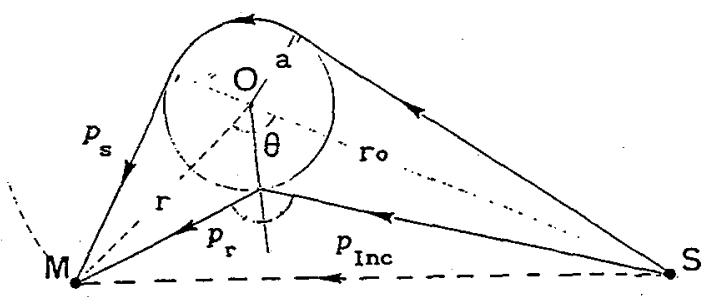

Figure 1 - Géométrie de l'expérience; trajets dans le plan équatorial.
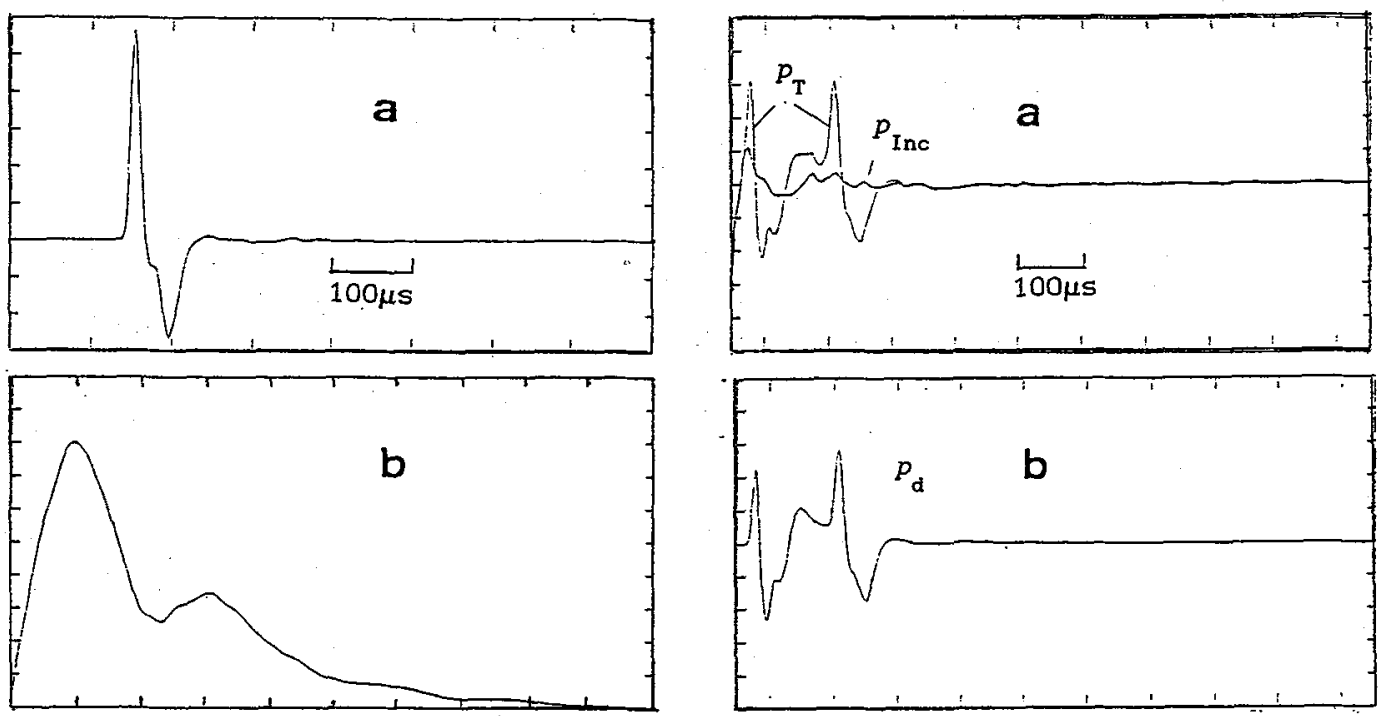

Figure 2 - Onde mesurée en $O(\theta=\Pi, r=0)$

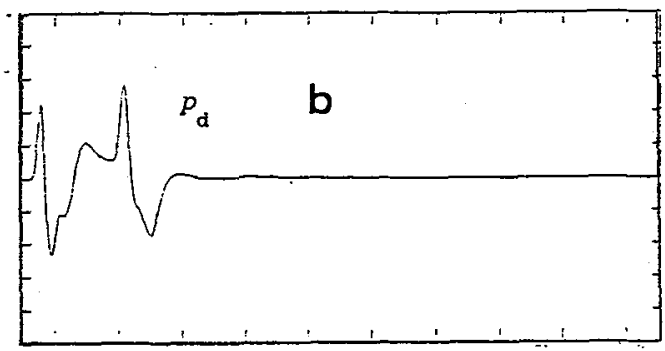

a. signal $p_{0}(t)$,

b. module du spectre $P_{0}^{\omega}[0,100 \mathrm{kHz})$.

Figure 3 - Cible $n^{*} 1, \theta=145^{\circ}$.

a. $p_{T}(t)$ et $p_{I n c}(t)$.

b. $P_{d}(t)=p_{T}(t)-p_{I n c}(t)$.

Le dispositif expérimental et la chaine de mesure utilisés [3,4] permettent d'effectuer la mesure des signaux en deux temps:

1. mesure de $I$ 'onde incidenté en 0 , sans cible $(\theta=\Pi, r=0): p_{0}(t)$ (figure 2a)

2. mesure de $I^{\prime}$ onde diffractée en $M(\theta, r): p_{d}(t)$

Elle se décompose en deux étapes:

- Mesure de I'onde "totale" en $M$ avec cible (figure $3 a) p_{T}(t)=p_{d}(t)+p_{I n c}(t)$.

. Mesure de l'onde "incidente" en $M$, cible otée (figure $3 a$ ) $p_{\text {Inc }}^{(t)}$.

On extrait 1'onde "diffractée" en $M$ (figure $3 b$ ): $p_{d}(t)=p_{T}(t)-P_{I n c}^{(t)}$.

Ensuite intervient une phase de traitement: Les signaux sont isolés dans des fenêtres temporelles $\Pi$ de durée $\mathrm{ri}$. Les spectres sont calculés par FFT:

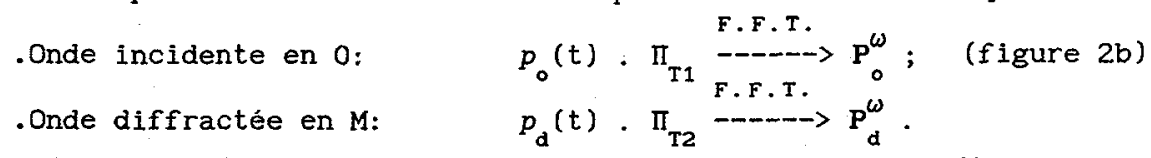

On en déduit la réponse en fréquences pour la diffraction en $M$ :

$$
\mathbf{R}_{1 \mathrm{M}}^{\omega}=\mathbf{P}_{\mathrm{d}}^{\omega} / \mathbf{P}_{0}^{\omega} . \quad \text { (figure 4) }
$$

Pour $\theta<\theta_{1 \mathrm{im}}$, l'onde diffractée se compose de "l'onde réfléchie" et de "l'onde rampante": $p_{d}(t)=p_{r}(t)+p_{s}(t)$. 
Si ces deux ondes sont séparées dans le temps, il est possible d'isoler "l'onde réfléchie" par filtrage temporel:

$$
P_{r}(t) . \pi_{T 3} \stackrel{F \cdot F \cdot T}{--m} P_{r}^{\omega} \text {, }
$$

et alors de calculer la réponse en fréquences pour la réflexion en $M$ :

$$
R_{2 M}=P_{r}^{\omega} / P_{0}^{\omega} \text {. (figure 4) }
$$

Les résultats sont, pour une géométrie donnée:

- le signal de l'onde diffractée,

- La réponse en fréquences de l'onde diffractée kae[0.1,15], et éventuellement celle de l'onde réfléchie.

Le diagramme de rayonnement pour une valeur choisie de $X=k a \quad(X \leq 15)$ peut être déduit des réponses en fréquences obtenues pour les différentes valeurs de $\theta$.

\section{CIBLE $n \cdot 1$, RESULTATS EXPERIMENTAUX.}

Les signaux diffractés en $M$ et les fonctions de transfert de la diffraction et de la réflexion (figure 4) sont obtenus pour différents angles $\theta$. Pour $\theta<\theta 1$ im, on note l'évolution de l'onde rampante $(\nabla)$ et de l'onde réfléchie. Des résultats assez semblables sont obtenus pour la cible $n^{\circ} 2$. Le filtrage temporel apporte une perturbation de la mesure de la fonction de transfert qui doit être limitée en BF (en particulier pour:l'étude du signal 'réfléchi' lorsque $\theta \simeq \theta l i m$ ).

Les diagrammes de rayonnement et du 'coefficient de réflexion' (figure 5) sont obtenus à partir de ces fonctions de transfert calculées pour des angles multiples de 5 degrés. A des fins de comparaison, les angles $\theta$ pour lesquels la différence de chemin $(\delta)$, dans le plan équatorial, entre l'onde 'Iéfléchie' et l'onde 'circonférentielle' est un multiple entier de longueur d'onde ( $\lambda$ ) sont localisés. sur le graphique.
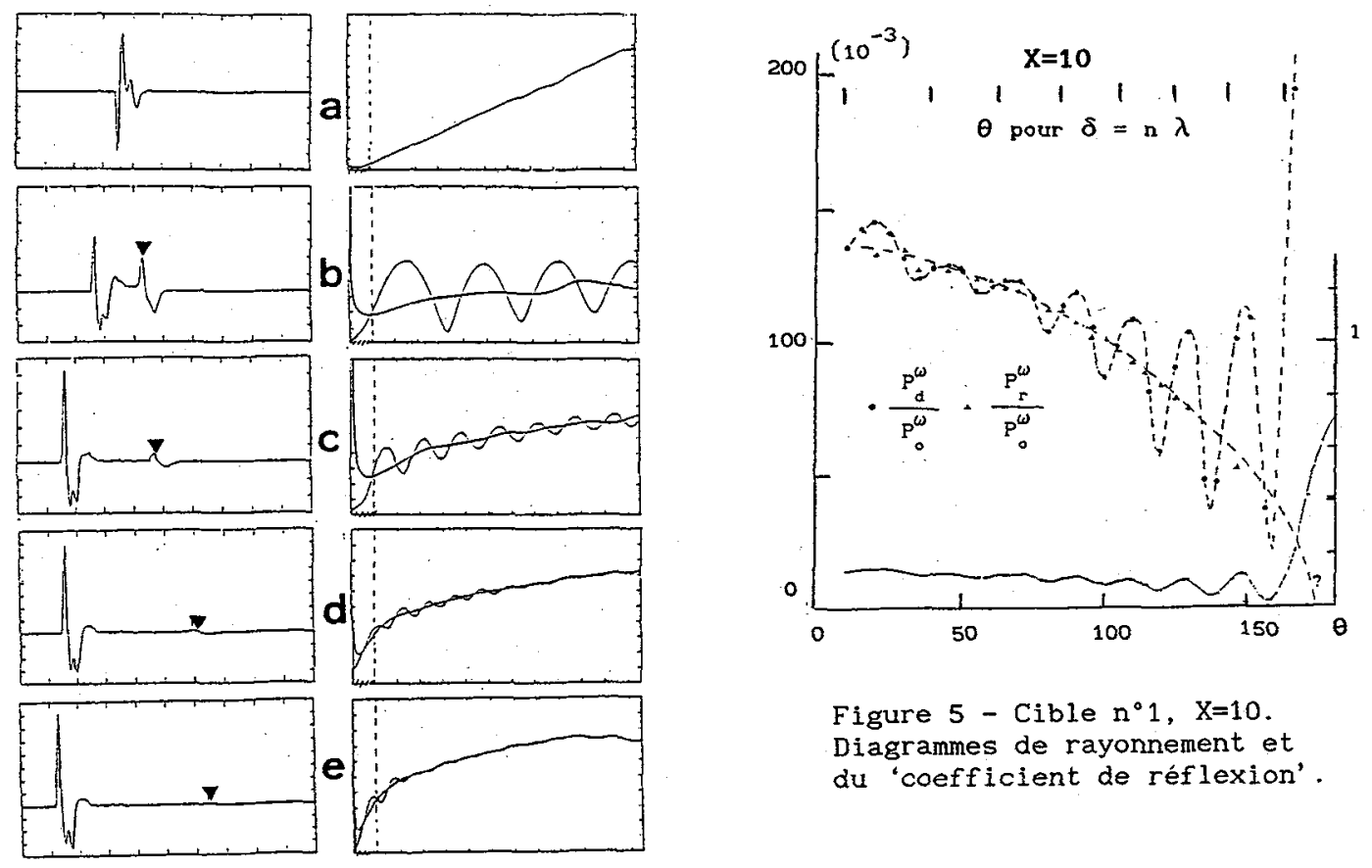

Figure 5 - Cible $\mathrm{n}^{\circ} 1, \mathrm{X}=10$. Diagrammes de rayonnement et du 'coefficient de réflexion'.

Figure 4 - Onde diffractée $p_{d}$, cible $n^{\circ} 1$, pour $\theta=a: 180^{\circ}, b: 135^{\circ}, c: 90^{\circ}, d: 45^{\circ}, e: 15^{\circ}$. signal $(5,6 \mathrm{~ms})$ et module correspondant de la réponse en fréquences (rayonnement et réflexion) $[0,25 \mathrm{kHz}]$. 
CIBLE $n^{\circ} 3$, COMPARAISON A LA CIBLE $n^{*} 1$.

Les mêmes mesures effectuées sur la cible de plus grand élancement ( $n^{\circ} 3$ ) permettent de séparer deux échos supplémentaires émanant des extrémités (figure 6 ). L'un (4), onde rampante au niveau des hémisphères, accompagne. I'onde circonférentielle (3) observée pour les autres cibles, l'autre (2), onde diffractée par les discontinuitées de courbure, s'intercale entre l'onde réfléchie (1) et l'onde circonférentielle (3). Les retards, presque constants, observés entre les échos $1-2$ et $3-4$ conforte $1^{\prime}$ attribution donnée. Pour $\theta=180^{\circ}$, une onde rampante ( $\nabla$ ) qui a parcouru,dans le plan vertical, un chemin tangent aux hémisphéres arrive également nettement détachée (figure 7). Sa présence se manifeste par une modulation quasi périodique de la réponse en fréquences.

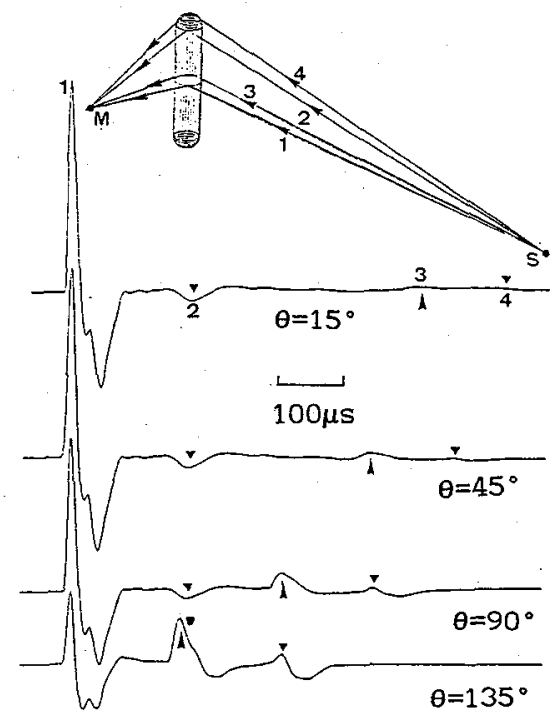

Figure 6 - cible $n^{\circ} 3$. géométrie des trajets et ondes diffractées pour différents $\theta$. (translation sur l'écho $n^{\circ} 1$ )
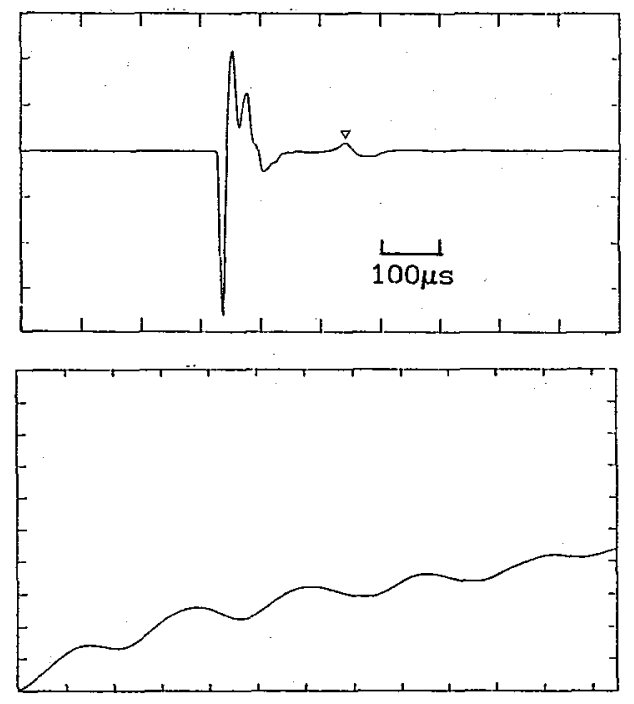

Figure 7 - ciblè $n^{\circ} 3 . \theta=180^{\circ}$. onde diffractée $p_{d}(5,6 \mathrm{~ms})$, module de la réponse en fréquences $(0,25 \mathrm{kHz})$.

\section{CONCLUSION.}

Ces mesures doivent permettre de vérifier globalement le calcul des diagrammes de rayonnement et du 'coefficient de réflexion'.

L'effet des terminaisons, bien que non directement observable sur les signaux de la cible $n^{\circ} 1$, est toujours présent et contribue à l'aspect parfois chaotique de la fonction de transfert représentant la diffraction (cibles $n^{\circ} 2$ et 3 ).

\section{REFERENCES.}

[1] BERGINC G., MOLINET F. Journal d'Acoustique 2 (1989) 19-29.

[2] KELLER J.B., Journal of Opt. Soc. of America $52 \mathrm{n}^{\circ} 2$ (1962) 116-130.

[3] DEPREZ G., HAZEBROUCK R. Acustica $45 \mathrm{n}^{\circ} 2$ (1980) 96-101.

[4] HAZEBROUCK $R$. Revue d'Acoustique $n^{\circ} 68$ (1984) 21-77. 